\title{
Erratum
}

\section{Self-assembled magnetic nanostructures: Epitaxial Ni nanodots on TiN/Si (001) surface}

\section{H. Zhou ${ }^{1,2 *}$ and J. Narayan ${ }^{1}$ hzhou@lanl.gov) \\ Erratum to: Journal of Nanoparticle Research DOI 10.1007/s11051-006-9098-1}

${ }^{1}$ Department of Materials Science and Engineering, North Carolina State University, Raleigh, NC, 27695 , USA; ${ }^{2}$ Superconductivity Technology Center, Materials Science \& Technology Division, Los Alamos National Laboratory, Los Alamos, NM, 87545, USA; *Author for correspondence (Tel.: + 64-3-364-2507; E-mail:

\section{H. Zhou and J. Narayan}

Superconducting Technology Center, Materials Science Technology Division, Los Alamos National Laboratory,Los Alamos, NM 87555,USA;*Author for correspondence (E-mail: hzhou@lanl.gov)

The above affiliations are incorrect. The affiliations below are correct:

H. Zhou* and J. Narayan

Department of Materials Science and Engineering, North Carolina State University, Raleigh, NC 27695, USA;*Author for correspondence (E-mail: hzhou@lanl.gov)

The corresponding author's current address is:

Superconductivity Technology Center, Materials Science \& Technology Division, Los Alamos National Laboratory, Los Alamos, NM 87545, USA.

The online version of the original article can be found at http://dx.doi.org/10.1007/s11051-006-9098-1 